\title{
Translation, cultural adaptation and validity of the Adolescent Sedentary Activity Questionnaire among school children aged 14-15 years
}

\author{
Indrani Godakanda ${ }^{1}$, Chrishantha Abeysena ${ }^{2 *}$, Ayesha Lokubalasooriya ${ }^{3}$ \\ ${ }^{1}$ Base Hospital, Panadura, Ministry of Health, Sri Lanka; ${ }^{2}$ Department of Public Health, Faculty of Medicine, \\ University of Kelaniya, Sri Lanka; ${ }^{3}$ Family Health Bureau, Ministry of Health, Sri Lanka
}

*Correspondence: chrishanthaabeysena@yahoo.com（iDhttps://orcid.org/0000-0001-5456-8281

DOI: https://doi.org/10.4038/jccpsl.v26i1.8192

Received on 22 January 2019

Accepted on 29 September 2019

\begin{abstract}
Introduction: Sedentary behaviour is a risk factor for several diseases. Validation of questionnaires on sedentary activity is a great challenge.

Objectives: To translate, culturally adapt and validate the Adolescent Sedentary Activity Questionnaire (ASAQ) among school children

Methods: Back translation method was used to translate ASAQ into Sinhala language (ASAQ-S). Cultural adaptation and judgmental validity were ensured using a panel of experts. Criterion validity was assessed by comparing ASAQ-S data with the reference standard, which was the ActiGraph GT1M accelerometer worn on the waist. The study population consisted of 42 school children aged 14-15 years. Sedentary activity time was taken from the accelerometer recorded data as total hours per day. The ASAQ-S has 14 items and then total sedentary time was calculated by summing up the activities of all seven days. Intra-class correlation coefficient (ICC) and 95\% confidence interval (CI) were calculated.
\end{abstract}

Results: The average sedentary time was 11.7 (SD=3.4) hours per day recorded by an accelerometer and from the ASAQ-S 9.0 ( $S D=2.3$ ) hours per day. An ICC for total sedentary activity time between the accelerometer and ASAQ-S was $0.52(95 \% \mathrm{CI}=0.08,0.78)$ and Pearson correlation coefficient was 0.55 ( $\mathrm{p}<0.01)$.

Conclusions: The validity of ASAQ-S was satisfactory and can be used as a tool to assess adolescent sedentary behaviour.

Key words: adolescence, cultural, questionnaire, reliability, sedentary, validity 


\section{Introduction}

The Adolescent Sedentary Activity Questionnaire (ASAQ) is dedicated to measure the time spent in a comprehensive range of sedentary activities by schoolaged children during out-of-school hours (leisure time) (1). This questionnaire identifies five sedentary categories and eleven normal weekday activities with one additional activity during the weekend in which adolescents were typically engaged. It covers comprehensive dimensions of sedentary activities and is designed to recall each of the past seven days of the week. It has well explained psychometric properties and is easy to administer. Students are asked to think about a normal week during the school term and to report how long they usually spend on each of these different sedentary activities before and after school (leisure time) during each day of the week for seven days (1).

Validating a sedentary activity questionnaire is a great challenge as it is a behaviour that is difficult to measure (2). Ideally, several validation procedures should be performed to establish the validity of an instrument (3). Judgmental validity assesses whether the conceptual definitions of sedentary behaviour has been effectively translated into operational terms and explained appropriately in operational definitions. This includes face validity, content and consensual validity (3). The face validity is a casual assessment of item appropriateness (4) that if a measure cannot satisfy this criterion, then the other criteria are inconsequential. Face validity is concerned with how appropriate, relevant and understandable the items in a questionnaire are to the focus or aim of the questionnaire (3). Content validity involves scrutinizing whether or not all aspects of measures under study were covered. Consensual validity is the use of agreement between two or more subject authorities as providing evidence for the validity of a phenomenon (3). ASAQ developers (1) recommended that when the ASAQ is used in other cultures, it must be modified accordingly and may need to be reviewed over time as other activities become popular.

The best and the most obvious way of appraising the validity of a questionnaire is by assessing the criterion validity; in which how well the measurement scale correlates with the reference standard is assessed. Accelerometer has been frequently used as criterion measures of sedentary and physical activity in validation studies (5-7). The objective of this study was to translate, culturally adapt and validate the ASAQ to assess the pattern of sedentary behaviour among school children.

\section{Methods}

\section{Translation of ASAQ}

Back translation method was used to translate the ASAQ into Sinhala language, while strictly adhering to rigorous translation and review procedures. Two translators fluent in both Sinhala and English languages independently translated the questionnaire to Sinhala. A third person fluent in both languages then compared the translations with the original English version. If there was disagreement, they were resolved through discussions with the translators, thereby yielding a provisional translation in order to select 'best fit' words and phrases. Next, the translators independently translated the provisional version back into English and compared with the original English version by an exercise expert, to ascertain whether the original meaning of the items had been retained. The differences were resolved by discussion with the translators and agreement was reached.

\section{Cultural adaptation}

We conducted two focus group discussions with 14-15 year old school children and their parents regarding the types of sedentary activities performed during children's leisure time (out-of-school hours) in order to confirm the relevance and completeness of the activities evaluated in the translated Sinhala questionnaire (ASAQ-S). In addition, suggestions were explored to recognize various variables and terminologies in relation to leisure time sedentary activities. ASAQ-S was then sent to a panel of expert reviewers comprising two education specialists and two public health specialists for their comments. The questionnaire was modified according to the suggestions that were gathered from the discussions with the adolescents and their parents. Finally, for pre-testing, ASAQ-S was administered to 20 school children aged $14-15$ years in the district outside the study area.

\section{Assessment of validity}

Validation of the ASAQ-S was carried out using judgemental validity followed by criterion validity.

The assessment of judgmental validity was made during the development phase by a panel comprising multi-disciplinary experts in the fields of public health, 
sports medicine and education. It was determined by assessing the agreement of experts on whether the conceptual definition has been translated appropriately in the tool and arriving at consensus.

For assessing the criterion validity of ASAQ-S against accelerometers, a descriptive cross-sectional study was carried out in five conveniently selected schools in Colombo District. The study population consisted of school children aged 14-15 years in Grades 9 and 10. The exclusion criteria were children with physical disabilities or any other history of chronic disease which restricts physical activities and children with writing and reading difficulties.

The required sample size was based on detecting a correlation coefficient of 0.5 for total time spent on sedentary behaviour during leisure time obtained from the accelerometer and ASAQ-S. This was based on validation studies conducted using the same equipment as the reference standard (15). With an alpha error of 0.05 and power of $90 \%$, the computed sample size was 40 . To obtain the sample, eligible students were handed over a sealed envelope that contained a consent form and a letter addressed to parents explaining the nature and purpose of the study and a description of the accelerometer. Of the 86 students who consented, 44 were selected randomly to participate in the study.

Data collection was done during a normal week of the school term exclusive of any extracurricular activities or examinations during this period. The reference standard used was the time spent on sedentary behaviour assessed by ActiGraph GTIM worn on the waist. The accelerometer summarizes data over a user specified time period, which is referred to as an "epoch" which ranges from 1-240 seconds. Accelerometers were set to record counts at 60 second epoch intervals. A number of previous studies have used one minute (8-9) or 30 second epochs (10). It has been suggested that when compared to adults, the sporadic nature of children's movements requires more frequent assessments (11). The cut-off point for threshold values of accelerometer counts was defined as $<100$ counts per minute for sedentary activities (10, 12-13). Continuous 20 minutes of zero counts per minute activity intensity period was considered as 'nonwearing' time (14). The use of accelerometers in estimating the sedentary activity of children and adolescents was justified since wearing an accelerometer does not interfere with normal daily activities of the participants (9).
Prior to data collection, the students and class teachers were instructed on how to wear the accelerometer followed by a demonstration. Accelerometers were then distributed to the students and were given a chance to test, in order to diminish the inquisitiveness about the instrument. Each child was asked to demonstrate the wearing of accelerometer. Proper instructions on how to complete the accelerometer diaries were also given.

Before using the accelerometer, the clock, day of the week and the unique identification number of the child were set. Students were instructed to wear the accelerometer for seven consecutive days while maintaining the diary as instructed. For weekends, students were instructed to wear the accelerometer from the time they woke up until they went to bed at night. They were asked to continue with their normal activities while wearing the accelerometers and instructed to remove during school hours, at sleep and before swimming, bathing and showering. They were instructed to mark the diary on removal and wearing times accurately for the purpose of cross-checking the accelerometer readings and diaries. The class teachers were requested to inspect each child's accelerometer to see that they were properly placed and secured with the safety belt and remind them to wear and remove the accelerometers every day, soon after they arrive at school and before leaving school. In addition, the parents were given further instructions for clarification. A trained research assistant visited the school twice a day to check their compliance.

Following the accelerometer assessment, administration of the ASAQ-S was done. ASAQ-S has been designed to recall the time spent on 14 different types of sedentary activity during each day of the past seven days. Students were asked to report how many hours and/or minutes they usually spent on each activity before and after school. Whenever adolescents marked two activities at the same time, for example, reading for pleasure in front of the TV - one hour, they were instructed to target periods of time spent on each activity, without doubling the time (e.g., 45 min television and 15 min reading).

\section{Reliability of ASAQ-S}

The test-retest reliability of the ASAQ-S was carried out in a randomly selected sample of 20 students in Grades 9 and 10. Following the administration of ASAQ-S, it was re-administered to the same students under the same conditions, two weeks apart. 


\section{Data analysis}

From the ASAQ-S data, total sedentary time per week was computed by summing up the time spent by each participant in all sedentary activities during weekdays and weekends.

Data from the ActiGraph accelerometer was downloaded to the ActiGraph computer software programme. Bouts of 20 continuous minutes of zero activity intensity counts were excluded from the analysis, considering these periods as non-wearing time. A minimum of six-hour period of wearing per day for at least three days of recording for weekdays and at least one day for the weekend (15) was set as an inclusion criterion and compared with diary recordings for completeness and accuracy of the data. Total sedentary time was expressed as total amount of time in minutes accumulated $<100$ counts per minutes (cpm) during periods when the accelerometer was worn. Total sedentary time was computed by summing up all seven days' sedentary time in minutes recorded from the accelerometer. Average sedentary time was computed by dividing the total sedentary time by the number of days.

Statistical analysis was performed using the Statistical Package for the Social Science (SPSS) version
16. Criterion validity was assessed by measuring interclass correlation coefficient (ICC) with 95\% CI between total minutes per week spent for sedentary behaviours (total sedentary time per week) obtained from the accelerometer and from the ASAQ-S. Reliability was assessed by calculating ICC with $95 \%$ CI between total sedentary minutes per week among the test group and retest group.

\section{Results}

During cultural adaptation, the question 'listening to radio' was included in the original version of ASAQ. The question 'using mobile or land phone' was placed as a separate variable while it was included under 'sitting around (chatting with friends/on the phone/ chilling)' in the original version and revised as 'stand/ sitting around, chatting with others'. Additionally, the time spent for drawing and painting was incorporated as a clarification in addition to the original question 'doing crafts or hobbies'. To the original question on 'travel (car/bus/train)', 'three-wheel taxi’ was added. The original question 'going to church or Sunday school was revised as 'spending at Sunday Dhamma school/ church/temple (weekends)' in the final version of the ASAQ-S (Table 1).

\section{Table 1. Items in the finalized ASAQ-S compared with the original ASAQ}

\begin{tabular}{ll}
\hline Original ASAQ & Finalized ASAQ-S \\
\hline - & Listening to radio \\
Watching TV & Watching TV \\
Watching videos/DVDs & Watching videos/DVDs \\
Using the computer for fun & Using the computer for fun \\
Using the computer for doing homework & Using the computer for doing homework \\
Doing homework not on the computer & Doing homework not on the computer \\
Reading for fun & Reading for fun \\
Being tutored & Being tutored/extra classes \\
Travel (car/bus/train) & Travel (car/three-wheeler/bus/train) \\
Doing crafts or hobbies & Doing crafts or hobbies, drawing, painting \\
Sitting around (chatting with friends/ & Stand/sitting around, chatting with others \\
on the phone/chilling) & \\
\multicolumn{1}{l}{ - } & Using the mobile or land phones \\
Playing/practising musical instruments & Playing/practicing musical instruments \\
Going to church or Saturday school & Spending at Sunday Dhamma school/church/temple (weekends) \\
\hline
\end{tabular}




\section{Criterion validity of ASAQ-S}

Analysis was performed using 42 children as two were excluded due to incompleteness of accelerometer data. Mean age was 14.5 years $(\mathrm{SD}=0.51)$. Majority of participants were Sinhalese (83.5\%) and $60 \%$ belonged to the rural school setting (Table 2). The mean body mass index (BMI) of boys was $18.9(\mathrm{SD}=3.13)$ and $18.84(\mathrm{SD}=3.11)$ for girls. BMI was normal in $68.2 \%$ of male and $60 \%$ of female participants, while $9.1 \%$ of males and $10 \%$ of females were overweight.

The total sedentary activity time was normally distributed. The average sedentary time was 11.7 $(\mathrm{SD}=3.4)$ hours per day recorded by accelerometer and $9.0(\mathrm{SD}=2.3)$ hours per day according to the ASAQ-S. An ICC for total sedentary activity time between the accelerometer and ASAQ-S was 0.52 $(95 \% \mathrm{CI}=0.08,0.78)$ and Pearson correlation coefficient was $0.55(\mathrm{p}<0.01)$. The mean sedentary hours per day for females were $11.9(\mathrm{SD}=3.4)$ compared to $11.6(\mathrm{SD}=3.6)$ in males according to accelerometer data. The mean sedentary hours per day for females were $9.6(+2.5)$ and $8.52(+1.9)$ were in males by ASAQ-S data.

\section{Reliability of ASAQ-S}

For total week sedentary time related to all activities, ICC showed 0.89 (95\% CI=0.71, 0.85) with statistically significant results (Table 3 ). The lowest ICC of 0.61 (95\% CI=0.24, 0.81) was observed for the item of playing/practising a musical instrument and the highest ICC of 0.98 (95\% CI=0.96, 0.99) was obtained for the item on tutored and extra classes.

Table 2. Socio-demographic and other characteristics of the study sample $(\mathrm{N}=42)$

\begin{tabular}{lcc}
\hline Characteristic & No. & \% \\
\hline Age & & \\
14 Years & 23 & 55.0 \\
15 Years & 19 & 45.0 \\
Sex & & \\
Male & 22 & 52.0 \\
Female & 20 & 48.0 \\
Ethnicity & & \\
Sinhalese & 35 & 83.5 \\
Tamil & 3 & 7.0 \\
Muslim & 4 & 9.5 \\
Sector & & \\
Urban & 17 & 40.0 \\
Rural & 25 & 60.0 \\
\hline
\end{tabular}

Table 3. Test-retest reliability of ASAQ-S

\begin{tabular}{lr}
\hline Sedentary activities (Total Week) & ICC (95\% CI) \\
\hline Listening to radio & $0.74(0.14,0.79)$ \\
Watching TV & $0.83(0.57,0.93)$ \\
Watching videos/DVDs & $0.76(0.38,0.9)$ \\
Using the computer for fun & $0.63(-0.18,0.81)$ \\
Using the computer for homework & $0.71(0.25,0.88)$ \\
Doing homework & $0.91(0.79,0.96)$ \\
Reading for fun & $0.7(0.26,0.88)$ \\
Being tutored/extra classes & $0.98(0.96,0.99)$ \\
Travel (car/ three-wheeler/bus/train) & $0.92(0.8,0.97)$ \\
Doing crafts or hobbies, reading, painting & $0.84(0.6,0.94)$ \\
Sitting around (chatting with friends/chilling) & $0.82(0.56,0.93)$ \\
Using the mobile or land phones & $0.78(0.43,0.91)$ \\
Playing/practising a musical instrument & $0.61(-0.24,0.81)$ \\
Spending at Sunday Dhamma school/church/temple & $0.95(0.87,0.97)$ \\
\hline Total sedentary behaviour time & $\mathbf{0 . 8 9} \mathbf{( 0 . 7 1 , 0 . 8 5 )}$ \\
\hline
\end{tabular}




\section{Discussion}

Results revealed that the ICC between the total sedentary time per week recorded by the accelerometer and ASAQ-S was 0.53, which showed moderate correlation. A previous study (16) reported a positive correlation of $0.63(\mathrm{p}<0.001)$ between the average percentage time spent per day in sedentary activities after school and weekend, measured by Physical Activity Questionnaire (PAQ) and the accelerometer among the age group of 9-11 years. Another study (17) found a significant correlation $(\mathrm{r}=0.48)$ between the proportion of time spent in sedentary behaviour $(<1.5$ METs) recorded from an interviewer-administered 24hour recall questionnaire and an accelerometer among middle school students. In contrast, Affuso (5) reported a weak correlation $(r=0.14)$ between the minutes of sedentary behaviour during past three days by PAQ and accelerometer among adolescence. Another study (18) reported ICC of 0.15 for a modified version of sedentary behaviour measure and accelerometer among 14-15-year old children. A short duration or limited activities assessment might not adequately capture the sedentary behaviours and may lead to the underestimation of the sedentary pursuits measured by accelerometer. The current study evaluated a wider range of sedentary pursuits through ASAQ-S for seven days.

The ASAQ-S showed $9.1(\mathrm{SD}=2.3$ ) hour per day of sedentary time spent for an average day. For the accelerometer data, it was $11.7(\mathrm{SD}=3.4)$ hours. It showed 2.6 hours of difference between ASAQ-S and accelerometer estimates of mean sedentary time per day. Another study (19) reported the mean weekly sedentary time difference between self-reported sedentary behaviour by ASAQ and accelerometer estimates was 3.2 hours per week among 12-15-year old girls. Two studies revealed that an underestimation of the self-reported minutes of sedentary behaviours (20-21) comparative to the accelerometer data in adolescents and adults. One study (21) stated that sedentary behaviours may be more difficult to remember than activities of higher intensity. Furthermore, total sedentary time accumulated by the accelerometer comprise all activities which meet the cut-off criterion for inactivity, and it includes activities which can occur incidentally during the course of a day, for example sitting without engaging in any activities, waiting in a queue and during an active sitting game. It is reasonable to assume that the recalls of these events are poor and therefore these episodes are not included in questionnaires, which seek to assess habitual behaviours (1). In addition, recall bias and social desirability can be associated with under reporting of sedentary behaviours with self-reported measures in adolescents (20), although it has made every effort to minimize any possible over- or under-reporting by the participants. In a longitudinal study (22) in Vietnam reported among adolescents aged 12-16 years, the average sedentary hours measured by accelerometers defining $<100$ cpm for sedentary was 7.4, 7.7, 8.3 and 8 hours per day respectively from 2005 to 2009 . According to a Canadian study (23), 11-14-year-old children spent 8.8 hours (8.7 for males and 8.8 for females) per average day engaging in sedentary behaviour using accelerometers.

In our study, the cut-off point of accelerometer counts (counts. minute 1) was defined as $100 \mathrm{cpm}$ for sedentary activities (9-10, 12-13). Cut-off points varying from 10 to $1592 \mathrm{cpm}$ have been proposed for defining sedentary time in children and adolescents in a number of accelerometers (24-25). Diversities in the selection of calibration, activities, criterion measures, statistical analyses and participant characteristics are likely to account for the variation in cut-off points anticipated to date (26). The three most commonly used cut-off points for sedentary behaviour among young people are 100, 800 and 1100 cpm (27).

In our study, bouts of continuous 20 minutes of zero cpm activity intensity period is considered as nonwearing time. In population-based studies, non-wear time is usually defined as periods of 10-60 minutes of consecutive zero counts, sometimes allowing for limited counts within these periods $(23,28)$.

\section{Reliability of ASAQ-S}

For the total week sedentary time related to all activities, the ICC showed 0.89 and for each activity ranging from 0.61 to 0.98 . The items of poorer reliability were 'surfing the internet for fun' and 'playing/practising a musical instrument'. Those behaviours depend upon other external factors such as academic chores and/or adolescents may not be able to estimate accurately the time span because these activities are short and sporadic. Some routinely engaged activities showed higher ICC such as 'being tutored or extra classes', 'doing homework', 'spending 
at Sunday Dhamma school' and 'travelling by car/three wheeler/bus/train'. This could be due to the stability of those activities over time rather than the reliability. Original questionnaire developers reported the testretest reliability (ICC) $\geq 0.7$ for total time spent in all sedentary behaviours among all categories of the ASAQ among school children in Australia. The reliability was generally lower for social activities and travel, except among high school girls on weekends. A study (29) reported the ICC for total time spent on sedentary behaviour by ASAQ as 0.88 for weekdays and 0.77 for weekends among Brazilian adolescents. The recall period was shorter than the present study. Another study in Europe (14) found that sedentary behaviours were less reliable during weekends (especially 'surfing the internet for study'), which was in agreement with the original questionnaire developers (1).

Sedentary activity is usually measured by selfadministered questionnaires in adolescents (26-27). Two systematic reviews suggest that self-report tools generally display acceptable validity in assessing sedentary behaviour (26-27). The major limitations of self- administered behavioural questionnaires are recall bias as well as response bias where respondents may intentionally provide incorrect answers to a survey due to pressure to respond in a socially acceptable manner $(20,30)$.

\section{Conclusions \& Recommendations}

The validity and the reliability of ASAQ-S were satisfactory and can be used as a tool to assess broad range of sedentary behaviours among the school children in Sri Lanka.

\section{Public Health Implications}

- Sedentary behaviour is a risk factor for several diseases.

- The reliability and the validity of the Sinhala version of the Adolescent Sedentary Activity Questionnaire (ASAQ-S) were satisfactory.

- It can be used as a tool to assess adolescent sedentary behaviour.

\section{Author Declarations}

Competing interests: The authors declare that they have no competing interests.

Ethics approval and consent to participate: The Ethics Review Committee of the Sri Lanka Medical Association granted ethical clearance. Written informed consent was obtained from parents and the school children prior to data collection. Permission was obtained from the relevant Provincial, District and Zonal Directors of Education, School Principals and Deputy Provincial Director of Health Services.

Funding: Self-funded

Acknowledgements: We are grateful to the Postgraduate Institute of Medicine, University of Colombo and the data collectors for the study.

Author contributions: IG, CA and IL conceptualized and designed the study. IG did the data collection and analysis and drafted the original manuscript. CA reviewed and edited the article. All authors read and approved the final manuscript.

\section{References}

1. Hardy LL, Booth ML, Anthony D, Okely AD. The reliability of Adolescent Sedentary Activity Questionnaire (ASAQ). Preventive Medicine 2007; 45(1): 71-74.

2. Rangul V, Holmen TL, Kurtze N, Cuypers K, Midthjell K. Reliability and validity of two frequently used selfadministered physical activity questionnaire in adolescents. BMC Medical Research Methodology 2008; 15: 8-47.

3. Abramson JH \& Abrahamson ZH. Survey Methods in Community Medicine ( $5^{\text {th }}$ edition). Toronto: Churchill Livinngstone,1999.

4. Litwin M. The Survey Kit: how to measure survey reliability and validity. California, volume 7, 1995.

5. Affuso O, Stevens J, Catellier D, McMurray R, Dianne $\mathrm{S}$, et al. Validity of self-reported leisure-time sedentary behaviour in adolescents. Journal of Negative Results in Biomedicine 2011; 10 :2.

6. Rosenberg DE, Bull FC, Marshall AL, Sallis JF, Bauman AE. Assessment of sedentary behaviour with the International Physical Activity Questionnaire. Journal of Physical Activity \& Health 2008; 5(Suppl. 1): S30-44. 
7. Karunapema RPR. Evaluation of the effectiveness of a lifestyle intervention in primary prevention of diabetes among adult with impaired fasting glucose from a rural area of Gampaha District. MD Thesis (Community Medicine). Colombo: Post Graduate Institute of Medicine, 2006.

8. Puyau MR, Adolph AL, Butte VNF. Validation and calibration of physical activity monitors in children. Obesity Research 2002; 10: 150-157.

9. Trost SG, Loprinzi PD, Moore R, Pfeiffer KA. Comparison of accelerometer cut points for predicting activity intensity in youth. Medicine \& Science in Sports \& Exercise 2011; 43(7): 1360-1368.

10. Treuth MS, Schmitz K, Catellier DJ, McMurray RG, Murray DM, et al. Defining accelerometer thresholds for activity intensities in adolescent girls. Medicine \& Science in Sports \& Exercise 2004; 36(7): 1259-1266.

11. Bailey RC, Olson J, Pepper SL, Porszasz J, Barstow TJ. The level and tempo of children's physical activities: an observational study. Medicine \& Science in Sports \& Exercise 1995; 27: 1033-1041.

12. Pulsford RM, Cortina-Borja M, Rich C, Kinnafick FE, Dezateux C, et al. Actigraph accelerometer-defined boundaries for sedentary behaviour and physical activity intensities in 7-year-old children. PLoS One 2011; 6(8): e21822.

13. Diouf A, Thiam M, Idohou-Dossou N, Diongue O, Mégné N, Diallo K, Sembène PM, Wade S. Physical activity level and sedentary behaviours among public school children in Dakar (Senegal) measured by PAQ$\mathrm{C}$ and Accelerometer: preliminary results. International Journal of Environmental Research and Public Health 2016; 10:13(10). pii: E998.

14. Moliner-Urdiales D, Ruiz JR, Ortega FB, Rey-Lopez JP, Vicente-Rodriguez G, et al. Association of objectively assessed physical activity with total and central body fat in Spanish adolescents; the HELENA Study. International Journal of Obesity 2009; 33: 1126-1135.

15. Craig CL, Marshall AL, Sjostrom M, Bauman AE, Booth ML, et al. International Physical Activity Questionnaire: 12 -country reliability and validity. Medicine and Science in Sports and Exercise 2003; 35(8): 1381-1395.

16. Nilsson A, Andersen LB, Ommundse Y, et al. Correlates of objectively assessed physical activity and sedentary time in children: a cross-sectional study (The European Youth Heart Study). BMC Public Health 2009; 9: 322.
17. Cradcock AL, Wiecha JL, Peterson KE, Sobol AM, Colditz GA, et al. Youth recall and TriTrac accelerometer estimates of physical activity levels. Medicine and Science in Sports and Exercise 2004; 36(3): 525-532.

18. Leatherdale ST, Laxer RE, Faulkner G. Reliability and validity of the physical activity and sedentary behaviour measures in the COMPASS study. COMPASS Technical Report Series. Waterloo, Ontario: University of Waterloo, 2014: 2(1).

19. Hardy LL, Bass SL, Booth ML. Changes in sedentary behaviour among adolescent girls: a 2.5-year prospective cohort study. Journal of Adolescent Health 2007; 40(2): 158-165.

20. Jago R, Baranowski T, Baranowski JC, Cullen KW, Thompson DI. Social desirability is associated with some physical activity, psychosocial variables and sedentary behaviour but not self-reported physical activity among adolescent males. Health Education Research 2007; 22(3): 438-449.

21. Buchowski MS, Townsend KM, Chen KY, Acra SA, Sun M. Energy expenditure determined by selfreported physical activity in related to body fatness. Obesity Research 1999; 7(1): 23-32.

22. Trang NHHD, Tang K, Hidde P, Van der P, Hardy LL, et al. Longitudinal sedentary behaviour changes in adolescents in Ho Chi Minh City. American Journal of Preventive Medicine 2013; 44(3): 223-230.

23. Colley RC, Garriguet D, Janssen I, Craig CL, Clarke J, Tremblay MS. Physical activity of Canadian children and youth: accelerometer results from the 2007 to 2009 Canadian Health Measures Survey. Health Reports 2011; 22:1-9.

24. Evenson KR, Catellier DJ, Gill K, Ondrak KS, McMurray RG. Calibration of two objective measures of physical activity for children. Journal of Sports Science 2008; 26: 1557-1565.

25. Krishnaveni GV, Mills IC, Veena SR, et al. Accelerometers for measuring physical activity behaviour in Indian children. Indian Paediatrics 2009; 46: 10551062.

26. Atkin AJ, Gorely T, Clemes SA, Yates T, Edwardson C, et al. Methods of measurement in epidemiology: sedentary behaviour. International Journal of Epidemiology 2012; 41: 1460-1471.

27. Schofield G, Brown R. Does sedentary behaviour contribute to chronic disease or chronic disease risk 
in adults? Scientific Committee of Agencies for Nutrition Action, July 2009. Available from: https:// ana.org.nz/wp-content/uploads/2016/11/Doessedentary-behaviour-contribute-to-chronic-diseaseor-chronic-disease-risk.pdf.

28. Healy GN, Wijndaele K, Dunstan DW, Shaw JE, Salmon J, et al. Objectively measured sedentary time, physical activity, and metabolic risk: the Australian Diabetes, Obesity and Lifestyle Study (AusDiab). Diabetes Care 2008; 31(2): 369-371.
29. Guimarães RF, Silva MP, Mazzardol ELO, Campoty WD. Reproducibility of Adolescent Sedentary Activity Questionnaire (ASAQ) in Brazilian adolescents. Revista Brasileira de Cineantropometria \& Desempenho Humano 2013; 15: 3.

30. Adams SA, Matthews CE, Ebbeling CB, Moore CG, Cunningham JE, et al. The effect of social desirability and social approval on self-reports of physical activity. American Journal of Epidemiology 2005; 161(4): 389-398. 